\title{
Ansiedad en niños y adolescentes debido a la cuarentena por COVID-19. Una encuesta en línea
}

\author{
Anxiety in children and adolescents due to COVID-19 quarantine. An online survey
}

\author{
Mariana Itzel López Garza, ${ }^{*}$ José Núñez del Prado, ${ }^{\ddagger}$ Priscilla Vázquez, ${ }^{\ddagger}$ \\ Edgar Alan Guillén Martínez, Eduardo Bracho Blanchet ${ }^{\Uparrow}$
}

Citar como: López GMI, Núñez del Prado J, Vázquez P, Guillén MEA, Bracho BE. Ansiedad en niños y adolescentes debido a la cuarentena por COVID-19. Una encuesta en línea. Acta Med GA. 2021; 19 (4): 519-523. https://dx.doi.org/10.35366/102539

\section{Resumen}

Objetivo: Encontrar la prevalencia actual de ansiedad en relación con la pandemia por COVID-19 en edad pediátrica de nuestro país y los factores que puedan estar asociados. Material y métodos: Estudio observacional, transversal, usando una encuesta en línea. Se incluyeron menores de siete a 17 años, cualquier sexo, mexicanos, que hayan vivido en México por lo menos dos semanas previas al llenado del cuestionario, participación voluntaria y encuestas completamente contestadas. Se excluyeron las encuestas que no cumplían con todos los criterios. Se realizaron estadísticas descriptivas y análisis comparativo. Se utilizó $\chi^{2}$, análisis de regresión logística bivariada y odds ratio para evaluar la relación entre variables y los factores predictores. Resultados: Novecientas setenta encuestas en total, 874 fueron analizadas. Relación significativa para mayor riesgo de presentar datos de ansiedad con el sexo femenino, contacto previo con un paciente COVID positivo, tener diagnóstico previo psicológico o psiquiátrico y falta de actividad física. Conclusiones: Es claro que los niños y adolescentes se vieron afectados en el área psicosocial debido a la cuarentena por COVID-19, lo que aumenta nuestro interés en su situación actual y nos lleva a iniciar un plan de acción para evitar complicaciones psicológicas/psiquiátricas en el futuro de nuestros pacientes.

Palabras clave: Ansiedad, niños, adolescentes, COVID-19, SARS-CoV-2.

\section{Abstract}

Objective: To find the current prevalence of anxiety related with the COVID-19 pandemic in the pediatric population in our country and the factors that may be involved. Material and methods: Observational cross-sectional study, using an online survey. Age between seven and 17 years old, any gender, Mexican nationality, who had lived in Mexico for at least two weeks prior to filling out the questionnaire, voluntary participation, and fully answered surveys were included. Surveys that did not meet all the criteria were excluded. Descriptive statistics and analysis were carried out. $\chi^{2}$, bivariate logistic regression analysis, and odds ratio were used to assess the relationship between the outcome variables and the predictor factors. Results: A total of 970 surveys, 874 were analyzed. Significant relationship for higher risk of having anxiety with female gender, previous contact with a positive covid patient, having a psychological or psychiatric diagnosis, and lack of physical activity. Conclusions: Children and adolescents were affected in this COVID-19 quarantine in their psychosocial area, which increases our interest in their current situation and leads us to initiate an action plan to avoid complications in the future of our patients.

Keywords: Anxiety, children, adolescents, COVID-19, SARS-CoV-2.
* Médico Residente de tercer año de Pediatría, Hospital Angeles Pedregal. Facultad de Medicina de la Universidad Nacional Autónoma de México. México.

₹ Pediatra, Neonatólogo. Servicio de Pediatría del Hospital Angeles Pedregal. Ciudad de México, México.

$\S$ Médico Residente de Cirugía General, Hospital Central Sur. Ciudad de México, México.

" Cirujano Pediatra. Servicio de Pediatría del Hospital Angeles Pedregal. Ciudad de México, México.
Correspondencia:

Dra. Mariana Itzel López Garza

Correo electrónico: mariana_loga9@live.com

Aceptado: 13-08-2021.

www.medigraphic.com/actamedica

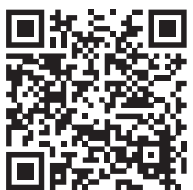




\section{INTRODUCCIÓN}

La emergencia sanitaria por el coronavirus 2019 (COVID-19) se declaró desde enero de 2020 a nivel mundial ${ }^{1}$ y en México desde el 30 de marzo del mismo año, ${ }^{2}$ la cual ha tenido repercusiones mundiales tanto en materia económica, social y política, como en materia de salud, física y mental, en todas las etapas de la vida. Entre las medidas para mitigar la dispersión del virus se implementó el distanciamiento social y el confinamiento, lo que llevó a la suspensión de actividades sociales, cierre de escuelas y centros de trabajo. En tiempos de pandemia los niños y adolescentes son especialmente vulnerables, donde tanto el propio crecimiento y desarrollo de los menores, los antecedentes familiares o personales, el ambiente, la frustración, el aburrimiento, la transformación de su estilo de vida, el miedo a infectarse, las condiciones de su vivienda, la falta de contacto con otras personas fuera de casa o la pérdida de algún ser querido causan repercusiones psicológicas en los individuos que se encuentran en estas etapas de la vida. ${ }^{3,4}$

En diferentes países del mundo se ha evidenciado que existe un impacto psicosocial significativo en niños y adolescentes a causa del aislamiento generado por la cuarentena por COVID-19 con un aumento en los niveles de ansiedad, principalmente en adolescentes. ${ }^{5-7}$ Con este trabajo buscamos encontrar la prevalencia de datos de ansiedad en personas en edad pediátrica de nuestro país, además de los factores que puedan estar implicados en ésta.

\section{MATERIAL Y MÉTODOS}

Se utilizó la aplicación de una encuesta en línea diseñada en formularios de Google y el método "bola de nieve", a partir de contactos cercanos de los autores que cumplían los criterios de inclusión. Es un estudio observacional de corte transversal. Se tomaron como criterios de inclusión la edad de entre siete y 17 años, cualquier sexo, nacionalidad mexicana, que hayan vivido en México por lo menos durante dos semanas previas al llenado del cuestionario, que participen voluntariamente, que proporcionen su consentimiento y que contesten completamente la encuesta. Se excluyeron aquellas encuestas que no fueron contestadas en su totalidad o que no cumplían con todos los criterios antes mencionados.

Cada encuesta constó de dos secciones: la primera sección dirigida a padres o tutores con el fin de recolectar datos demográficos sobre los participantes y su familia durante la cuarentena. La segunda sección incluye un cuestionario para identificar datos de ansiedad en niños y adolescentes mexicanos.

Para evaluar la ansiedad se utilizó la escala validada al español SCARED (Screen for Child Anxiety Related Emotio- nal Disorders) diseñada como una herramienta de tamizaje de los trastornos de ansiedad, consta de 41 reactivos, con tres opciones de respuesta (de 0 a 2 puntos), se tomó como punto de corte 25 puntos, referido por la literatura con una probabilidad de al menos $70 \%$ de que el niño o adolescente tenga algún trastorno de ansiedad. Esta encuesta está recomendada para identificar los trastornos de ansiedad en niños y adolescentes, como una herramienta de utilidad para el profesional de la salud, no psiquiatra, ya que permite discernir si un menor de edad ameritará el envío a tratamiento psiquiátrico o no. Sin embargo, no sustituye el diagnóstico clínico.

La encuesta fue contestada previo consentimiento del padre o tutor del menor y asentimiento del menor, para lo cual se le pidió que marcara las casillas correspondientes en el formulario donde aceptaba participar de manera voluntaria tanto él/ella como su hijo, así como haber leído y entendido la información proporcionada previo al Ilenado de la encuesta, lo que fue tomado como consentimiento informado. Se explicó, aparte del objetivo de la investigación, que su participación era totalmente anónima y que tanto el padre o tutor como su hijo eran libres de terminar su participación en el estudio en cualquier momento, además de que no recibirían ningún tratamiento ni se les haría ningún procedimiento clínico. Se les solicitó de manera opcional un correo electrónico. La información recolectada, según la Ley Federal de Protección de Datos Personales en Posición de Particulares, fue controlada por el propio usuario y salvaguardada por los investigadores.

Se analizaron las siguientes variables: ansiedad, según el punto de corte establecido y los factores predictores relevantes (edad, sexo, compañía durante el día, trabajo de los padres, disminución de ingresos, oportunidad de salir al aire libre en casa, mascotas, hermanos, horarios o rutinas establecidas en casa, disponibilidad y duración de entretenimiento electrónico, actividad física regular, contacto directo con una persona positiva a COVID-19, horas pantalla totales para actividades académicas y de entretenimiento y antecedentes psicológicos o psiquiátricos). Se realizaron estadísticas descriptivas y análisis de la frecuencia de las características demográficas. Para el análisis univariado de asociación de variables con datos de ansiedad se utilizó $\chi^{2}$, posteriormente se realizó análisis multivariado mediante regresión logística múltiple bivariada, calculando odds ratio y sus intervalos de confianza al 95\% (IC 95\%). El nivel de significancia estadística establecida fue de 0.05.

\section{RESULTADOS}

Se contestaron un total de 970 encuestas, 874 (90.1\%) reunieron los criterios de inclusión y fueron tomadas en 
consideración para su análisis. Se excluyeron 96 encuestas por diversos criterios de exclusión.

De los 874 participantes, 547 (62.6\%) corresponden al sexo femenino y $327(37.4 \%)$ al masculino. Se dividió la muestra en dos rangos de edad, de siete a 11 años y de 12 a 17 años, siendo el grupo de mayor edad quienes mostraron una mayor participación con $71.6 \%$ del total de las encuestas.

Del total de los padres de familia encuestados $73.7 \%$ tuvieron disminución en sus ingresos mensuales a causa de la pandemia. El 10\% aceptó haber perdido su empleo a causa de la cuarentena y $66.8 \%$ mencionó trabajar actualmente, de éstos, $49.3 \%$ salían a trabajar para cubrir sus jornadas laborales, $12.4 \%$ realizaron solo trabajo en casa (home office) y $7.1 \%$ son trabajadores del sector salud. El 23.2\% restante se dedican al cuidado de sus hijos y el hogar. El 3.7\% mencionó que sus hijos se quedan sin compañía durante el día y, de ellos, 75\% presentó datos de ansiedad.

El 53\% de nuestros encuestados mencionó que su hijo, además de las horas dedicadas a las actividades escolares, pasa más de dos horas frente a cualquier pantalla como entretenimiento y $24.1 \%$ del total dedica más de seis horas diarias a la actividad escolar en línea.

Se identificó un total de 563 casos que presentan datos de ansiedad, lo cual representa $64.4 \%$ de la población encuestada. Con el análisis univariado, $\chi^{2}$ y odds ratio (OR) encontramos que los factores asociados a ansiedad fueron el sexo femenino (OR: 2.165, IC: 1.624, 2.880), el grupo de edad de 12 a 17 años (OR: 1.853, IC: 1.317, 2.504), el contacto previo con un paciente COVID positivo (OR: 1.705, IC: 1.255, 2.316), tener algún antecedente psicológico o psiquiátrico establecido (diagnósticos no especificados en nuestra encuesta) (OR: 5.735, IC: 3.479, 9.454), el uso de más de seis horas de pantalla para tareas escolares (OR: 1.580, IC: 1.125, 2.217) y más de dos horas para entretenimiento electrónico (OR: 1.448, IC: 1.097, 1.912). Como factores protectores se encontró la actividad física regular (OR: 0.686, IC: 0.686, 0.906) y tener horarios establecidos (OR: 0.663, IC: 0.500, 0.880). Posterior a la regresión logística binaria obtuvimos que las variables con significancia como factor predictor para tener datos de ansiedad fueron pertenecer al sexo femenino, no tener actividad física, contacto previo con un paciente afectado por COVID-19 y tener algún antecedente psicológico o psiquiátrico establecido (Tablas 1 y 2).

\section{DISCUSIÓN}

Este estudio encontró un mayor número de individuos en edad pediátrica con ansiedad en comparación con otros estudios de diferentes partes del mundo. ${ }^{6,8-10}$
Se encontró que la prevalencia de ansiedad fue casi dos veces mayor en el sexo femenino como lo mencionan otros autores, dicho riesgo se ha publicado hasta de tres veces más, sobre todo en las adolescentes. ${ }^{6,8-13}$

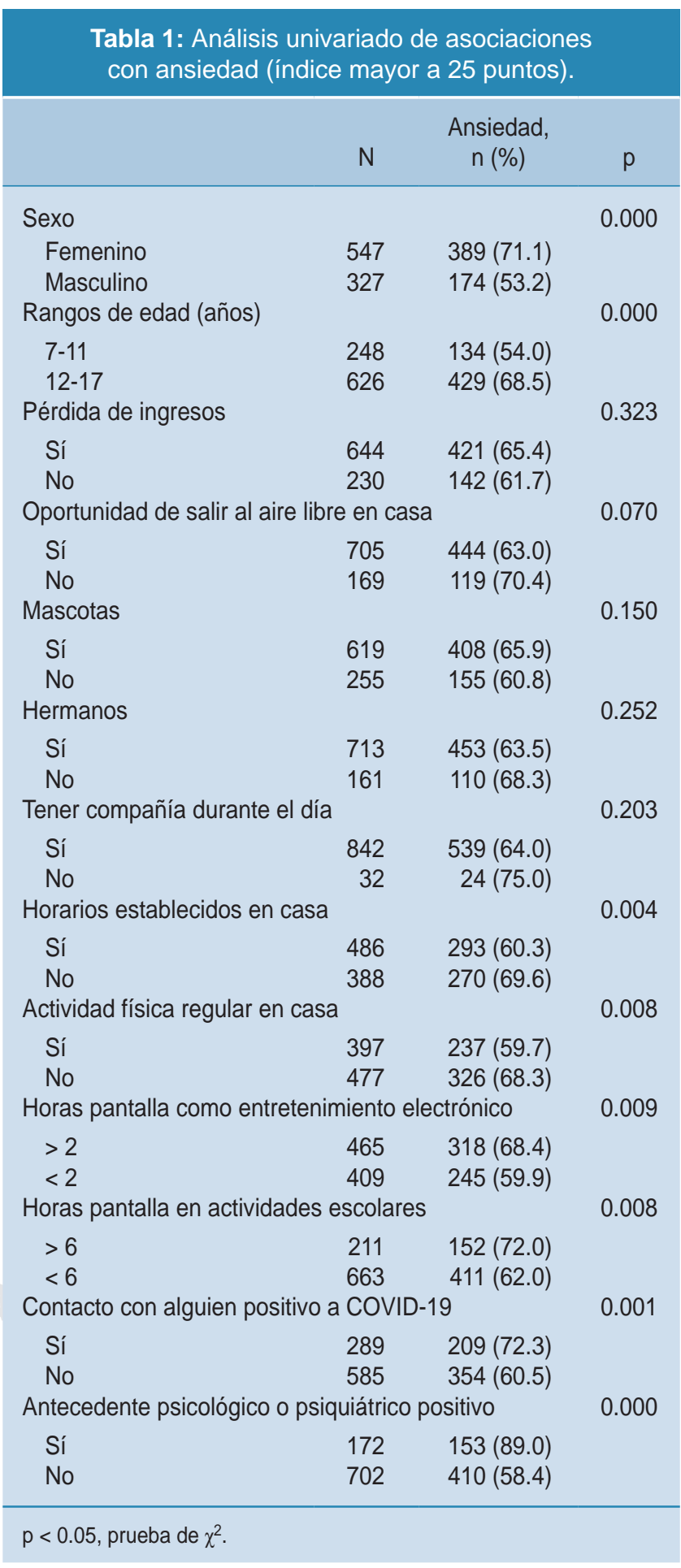




\begin{tabular}{|c|c|c|}
\hline Variable & OR & IC 95\% \\
\hline Sexo femenino & 1.925 & $1.423-2.605$ \\
\hline No tener actividad física regular & 1.385 & $1.027-1.867$ \\
\hline Contacto con alguien positivo a COVID-19 & 1.413 & $1.020-1.957$ \\
\hline Antecedente psicológico o psiquiátrico positivo & 4.769 & $2.858-7.960$ \\
\hline
\end{tabular}

El estar relacionado directamente con un contacto infectado con COVID-19 fue un factor para el aumento de datos de ansiedad en la población, como es mencionado por un estudio en China ${ }^{14}$ y confirmado en nuestro estudio. México se posicionó dentro de los primeros 10 lugares en el mundo por muertes de COVID-19. ${ }^{15}$ Lo cual puede tener un efecto negativo en la salud mental de la sociedad y de quienes están en contacto, ya sea con casos positivos, casos sospechosos y/o muertes por COVID-19.

Existe una relación establecida entre ansiedad y los individuos con antecedentes psicológicos o psiquiátricos, ya que estos jóvenes son propensos a presentar episodios de preocupación por familiares o por ellos mismos, de enfermar o morir a causa del virus, además de encontrarse en una dinámica familiar afectada por la cuarentena, y si antes podían distraerse con amigos o actividades recreativas, el confinamiento aumentó sus preocupaciones. 4,16,17 Nosotros encontramos esta misma relación, sin embargo, no es posible demostrar si existe o no asociación con algún padecimiento específico, debido a que en nuestra encuesta no establecimos el diagnóstico de los participantes por cuestiones de confidencialidad.

Otro factor relacionado con aumento en los datos de ansiedad fue el no tener actividad física regular, está descrito que la actividad física ayuda a disminuir los niveles de ansiedad durante periodos de estrés, ${ }^{18}$ beneficio que no se presenta en estos niños y adolescentes; además los padres de familia encuestados reportaron un uso mayor a dos horas de pantallas como entretenimiento en 53\% de los casos, y de éstos $68 \%$ presentaron datos de ansiedad, esto, aunque no fue significativo en la regresión multivariada, debe tomarse en cuenta, ya que los menores de edad también utilizan los dispositivos electrónicos para actividades escolares, uso que en nuestra población fue mayor a seis horas en $24 \%$ de los casos, relacionándose también con mayores niveles de ansiedad.

Este aumento en las horas pantalla también aumenta el tiempo de sedentarismo, que reduce la actividad física y la exposición a la luz solar, lo cual afecta la homeostasis del sueño y lleva a un mayor riesgo de padecer ansiedad. Es bien sabido que el insomnio se volvió un problema frecuente en esta cuarentena debido al aumento del estrés en la población por preocupaciones financieras, de salud o los cambios presentados en las relaciones sociales o actividades de la vida diaria, los cuales afectaron los horarios habituales. ${ }^{18-20}$

El grupo de edad más afectado fue el mayor de 12 años con más de $60 \%$ de los adolescentes afectados, es importante mencionarlo, ya que ha sido demostrado en diversos estudios que los adolescentes son una población vulnerable en tiempos de pandemia para padecer problemas de salud mental, con una prevalencia mayor de ansiedad que ha sido reportada entre 28.3 y $49.7 \%$, un rango menor a lo encontrado en nuestra muestra, aunque no mostró diferencia estadística en la regresión multivariada. El encierro por COVID-19, la pérdida del contacto con personas fuera de casa y la pérdida de las clases presenciales representan un factor de riesgo importante para este grupo poblacional. $6,9,10,13,16$

La relación entre no tener compañía durante el día y los altos niveles de ansiedad fueron mencionados en un estudio en China; ${ }^{21}$ nosotros encontramos que $75 \%$ de los niños y adolescentes que se encontraban en esta situación presentaron datos de ansiedad; sin embargo, no fue estadísticamente significativo cuando se analizó la relación entre variables, esto probablemente por el bajo número de participantes en esta condición.

Se observó que $73.7 \%$ de los padres de familia disminuyeron sus ingresos mensuales, sin un incremento en los niveles de ansiedad de sus hijos en relación con las familias que no disminuyeron sus ingresos.

En México inició la cuarentena desde marzo de 2020 y en la actualidad empieza a haber cambios en las medidas utilizadas para la contención de la pandemia, ${ }^{22}$ como el plan para iniciar las clases presenciales y con esto comenzar nuevamente la interacción de los niños y adolescentes en un plantel educativo. Esto nos lleva a dar un seguimiento de 
nuestros pacientes en edad pediátrica para poder identificar si existe una continuidad de los síntomas u otros problemas psicosociales, ya que se ha reportado en pasadas contingencias que las experiencias adversas que se viven en la infancia pueden afectar a largo plazo a los supervivientes. ${ }^{23}$

Después de nuestro estudio recomendamos que se hable de manera adecuada y de acuerdo con su edad a los menores sobre el tema, que se permita expresar emociones, ideas o miedos sobre la situación que viven, mantener una comunicación constante con las personas cercanas que no viven en casa, ya sea por videollamadas, mensajes de texto o llamadas regulares, diseñar y tener un plan diario con horarios establecidos principalmente a la hora de ir a la cama, las tareas escolares y el uso de dispositivos electrónicos, regular y vigilar adecuadamente el uso de pantalla al día, realizar actividad física, mantener actividades al aire libre en medida de lo posible, aumentar el tiempo en familia con actividades que permitan fomentar la comunicación, la responsabilidad, la colaboración y la participación de cada miembro del hogar.

\section{REFERENCIAS}

1. Mahase E. China coronavirus: $\mathrm{WHO}$ declares international emergency as death toll exceeds 200. BMJ [Internet]. 2020; 368: m408. Available in: http://dx.doi.org/doi:10.1136/bmj.m408

2. Consejo de Salubridad General declara emergencia sanitaria nacional a epidemia por coronavirus COVID-19 | Secretaría de Salud | Gobierno | gob.mx [Internet]. [cited 2021 May 5]. Disponible en: https://www.gob.mx/salud/prensa/consejo-de-salubridad-generaldeclara-emergencia-sanitaria-nacional-a-epidemia-por-coronaviruscovid-19-239301

3. Wang G, Zhang Y, Zhao J, Zhang J, Jiang F. Mitigate the effects of home confinement on children during the COVID-19 outbreak. Lancet. 2020; 395 (10228): 945-947.

4. Sánchez Boris IM. Impacto psicológico de la COVID-19 en niños y adolescentes. MEDISAN [Internet]. 2021; 25 (1): 123. Disponible en: http://medisan.sld.cu/index.php/san/article/view/3245

5. Tang F, Liang J, Zhang H, Kelifa MM, He Q, Wang P. COVID-19 related depression and anxiety among quarantined respondents. Psychol Heal [Internet]. 2021; 36 (2): 164-178. Available in: https://doi.org/10.10 80/08870446.2020.1782410

6. Liu J, Zhu Q, Fan W, Makamure J, Zheng C, Wang J. Online mental health survey in a medical college in China during the COVID-19 outbreak. Front Psychiatry. 2020; 11: 459.

7. Mazza C, Ricci E, Biondi S, Colasanti M, Ferracuti S, Napoli C et al. A nationwide survey of psychological distress among Italian people during the COVID-19 pandemic: immediate psychological responses and associated factors. Int J Environ Res Public Health [Internet]. 2020; 17 (9): 3165. Available in: https://www.ncbi.nlm.nih.gov/pmc/articles/ PMC7246819/pdf/ijerph-17-03165.pdf

8. Ahmed MZ, Ahmed O, Aibao Z, Hanbin S, Siyu L, Ahmad A. Epidemic of COVID-19 in China and associated psychological problems. Asian
J Psychiatr [Internet]. 2020; 51: 102092. Available in: https://doi. org/10.1016/j.ajp.2020.102092

9. Xie X, Xue Q, Zhou Y, Zhu K, Liu Q, Zhang J et al. Mental health status among children in home confinement during the coronavirus disease 2019 outbreak in Hubei province, China. JAMA Pediatr. 2020; 174 (9): 898-900.

10. Zhou SJ, Zhang LG, Wang LL, Guo ZC, Wang JQ, Chen JC et al. Prevalence and socio-demographic correlates of psychological health problems in Chinese adolescents during the outbreak of COVID-19. Eur Child Adolesc Psychiatry [Internet]. 2020; 29 (6): 749-758. Available in: https://doi.org/10.1007/s00787-020-01541-4

11. Wang Y, Di Y, Ye J, Wei W. Study on the public psychological states and its related factors during the outbreak of coronavirus disease 2019 (COVID-19) in some regions of China. Psychol Heal Med. 2021; 26 (1): 13-22.

12. Ozdin S, Bayrak Ozdin S. Levels and predictors of anxiety, depression and health anxiety during COVID-19 pandemic in Turkish society: the importance of gender. Int J Soc Psychiatry. 2020; 66 (5): 504-511.

13. Marques de Miranda D, da Silva Athanasio B, Sena Oliveira AC, Simoes-E-Silva AC. How is COVID-19 pandemic impacting mental health of children and adolescents? Int J Disaster Risk Reduct. 2020; 51: 101845

14. Cao W, Fang Z, Hou G, Han M, Xu X, Dong J et al. The psychological impact of the COVID-19 epidemic on college students in China. Psychiatry Res [Internet]. 2020; 287: 112934. Available in: https:// doi.org/10.1016/j.psychres.2020.112934

15. Forbes Staff. México, lugar 10 en el mundo por muertes de COVID-19: Salud [Internet]. [Citado 7 de mayo de 2021]. Disponible en: https://www.forbes.com.mx/noticias-mexico-decimo-lugarmundo-muertes-covid-19-salud/

16. Guessoum SB, Lachal J, Radjack R, Carretier E, Minassian S, Benoit L et al. Adolescent psychiatric disorders during the COVID-19 pandemic and lockdown. Psychiatry Res [Internet]. 2020; 291: 113264. Available in: https://doi.org/10.1016/j.psychres.2020.113264

17. Palacio-Ortiz JD, Londoño-Herrera JP, Nanclares-Márquez A, Robledo-Rengifo P, Quintero-Cadavid CP. Trastornos psiquiátricos en los niños y adolescentes en tiempo de la pandemia por COVID-19. Rev Colomb Psiquiatr. 2020; 49 (4): 279-288.

18. Alves JM, Yunker AG, DeFendis A, Xiang AH, Page KA. Associations between affect, physical activity, and anxiety among US children during COVID-19. medRxiv. 2020.

19. Voitsidis P, Gliatas I, Bairachtari V, Papadopoulou K, Papageorgiou G, Parlapani E et al. Insomnia during the COVID-19 pandemic in a Greek population. Psychiatry Res [Internet]. 2020; 289: 113076. Available in: https://doi.org/10.1016/j.psychres.2020.113076

20. Becker SP, Gregory AM. Editorial perspective: perils and promise for child and adolescent sleep and associated psychopathology during the COVID-19 pandemic. J Child Psychol Psychiatry. 2020; 61 (7): 757-759.

21. Chen F, Zheng D, Liu J, Gong Y, Guan Z, Lou D. Depression and anxiety among adolescents during COVID-19: A cross-sectional study. Brain Behav Immun. 2020; 88: 36-38.

22. Semáforo - Coronavirus [Internet]. [Citado 5 de mayo de 2021]. Disponible en: https://coronavirus.gob.mx/semaforo/

23. Fegert JM, Vitiello B, Plener PL, Clemens V. Challenges and burden of the Coronavirus 2019 (COVID-19) pandemic for child and adolescent mental health: a narrative review to highlight clinical and research needs in the acute phase and the long return to normality. Child Adolesc Psychiatry Ment Health [Internet]. 2020; 14: 20. Available in: https://doi.org/10.1186/s13034-020-00329-3 